\title{
modèle viscoplastique pour le comportement d'un tunnel revêtu
}

\author{
par \\ P. Bérest \\ D. Nguyen Minh \\ Laboratoire de Mécanique des Solides \\ (E.P., E.N.P.C., E.N.S.M.) associé au C.N.R.S.
}

\section{Introduction}

La convergence observée dans les tunnels et galeries souterraines est la plupart du temps un phénomène différé, dont les manifestations peuvent persister plusieurs semaines ou plusieurs mois après le creusement et même la pose d'un revêtement. Panet [1979] a discuté les modèles de calcul qui permettent de rendre compte de ces phenomènes : la plupart de ces modèles sont du type viscoélastique, de sorte que leur champ d'application est limité à un nombre assez faible de situations réelles. On propose un modèle de calcul des effets du creusement et de la pose d'un revêtement qui tienne compte d'un comportement viscoplastique du massif. II est ainsi possible de discuter l'influence de la viscosité sur les résultats obtenus par la méthode de convergence-confinement (Tunnels et Ouvrages Souterrains [1979]) qui se place dans le cadre d'un comportement élastoplastique des terrains.

\section{Hypothèses du calcul}

On considère un tunnel de section circulaire suffisamment long pour qu'on puisse traiter le comportement d'une section comme un problème en déformation plane. Le poids propre des terrains est négligé; à grande distance de la paroi règne un état de contrainte isotrope $\sigma_{j \mathrm{k}}=-\mathrm{P}_{\infty} \delta_{\mathrm{j} \mathrm{k}}$ i à la paroi est appliquée une contrainte normale $\sigma_{i}$. Sous ces hypothèses les grandeurs mécaniques définies dans le massif ne dépendent que de la coordonnée $r$. On prend le rayon de la galerie comme unité de longueur.

Le comportement du massif est viscoplastique, du type dit de Bingham; le critère viscoplastique ne dépend que de l'écart entre les contraintes principales extrémales (critère de Tresca).

Du fait du caractère visqueux du comportement, I'histoire du chargement doit être décrite avec soin. On suppose qu'à l'instant initial la contrainte normale à la paroi est instantanément portée à une valeur nulle: autrement dit, la durée nécessaire au creusement du tunnel est négligée. Puis, après un certain intervalle de temps pendant lequel la convergence a commencé à progresser, on pose un revêtement élastique sur la paroi : la contrainte normale à la paroi cesse alors d'être nulle, on appelle sa valeur absolue "pression de revêtement "; du fait du caractère élastique du revêtement, cette contrainte est liée linéairement à l'accroissement de convergence après la pose du revêtement.

Un des problèmes les plus importants du point de vue pratique est de connaitre la valeur ultime que prendra la pression de revêtement: le calcul présenté plus loin permet de plus de décrire comment les différentes grandeurs convergent vers leur valeur finale; mais on mettra surtout l'accent sur la détermination de l'état ultime.

Remarque sur les rapports entre le modèle retenu et le comportement d'un tunnel réel.

Les hypothèses introduites constituent une simplification notable des situations réelles, du point de vue de la géométrie et de l'influence propre de la gravité; la discussion de la validité de ces simplifications sort du cadre du présent article. De même, le comportement viscoplastique retenu est assez particulier et ne peut représenter valablement que des roches assez molles. comme l'argile, le sel ou les marnes; mème pour ces matériaux, la loi retenue n'est sans doute qu une première approche d'un comportement rhèologique plus complexe; néanmoins, cette loi constitue un progrès sensible par rapport à des modèles plus traditionnels, qui ne prennent en compte qu'un comportement élastoplastique sans effets différés ou des comportements élastiques à effets différés.

Enfin, on a délibérément fait le choix de négliger un aspect important de l'histoire du chargement, qui est la mise en charge progressive du tunnel consecutive au passage puis à l'éloignement du front de creusement Cette mise en charge progressive est pourtant un 


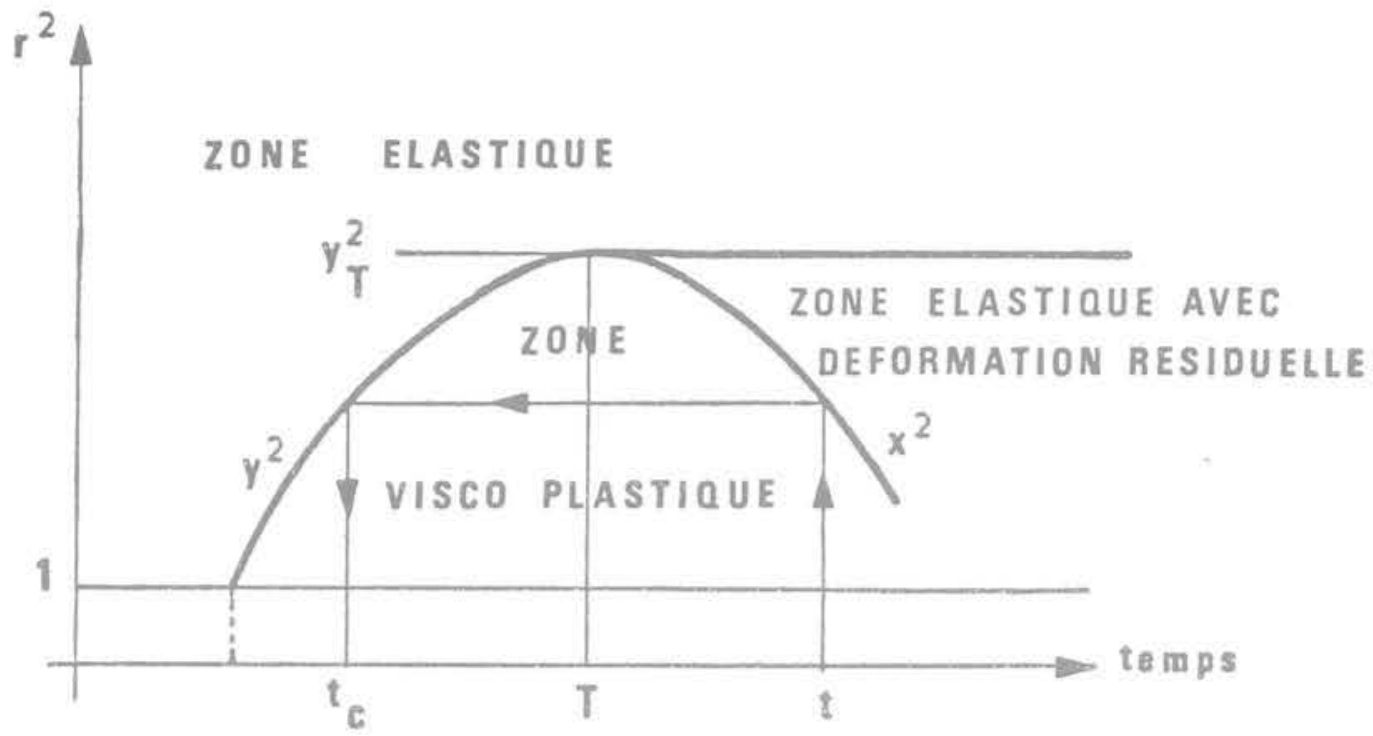

Fig. 1 Développement en retrait d'une zone viscoplastique

élément important du comportement, qui joue un rôle essentiel dans l'interprétation de la méthode dite de "convergence-confinement". On doit faire à ce propos plusieurs remarques:

- L'objet du présent article est de mettre en évidence un phénomène particulier, qui est le rôle propre de la viscosité du comportement du massif. Le calcul ne vise donc pas d'abord à interpréter les convergences d'un tunnel réel mais à construire un modèle qui permet d'évaluer un aspect du comportement. Introduire une mise en charge progressive dans ce calcul ne pose aucun problème de principe; le lecteur vérifiera simplement que les formules en seraient un peu compliquées, sans intérêt particulier pour le but visé.

- Néanmoins, le calcul présenté conserve un intérêt pratique immédiat lorsque le massif présente une forte viscosité, ou plus précisément lorsque le temps après lequel l'influence du front est négligeable est petit visà-vis du temps de relaxation caractéristique du comportement visqueux du massif:

\section{Conduite du calcul}

Les hypothèses formulèes plus haut conduisent au système d'équations :

(1) $E \frac{\partial u}{\partial r}=\sigma_{r}-\nu\left(\sigma_{\theta}+\sigma_{z}\right)+E \varepsilon_{v p}+E \varepsilon_{v p}+(1-2 \nu) p_{x}$

(2) $E \frac{U}{r}=\sigma_{\theta}-v\left(\sigma_{r}+\sigma_{z}\right)-E \varepsilon_{v \rho}+(1-2 v) P_{\infty}$

(3) $\varepsilon_{z}=0=\sigma_{z}-v\left(\sigma_{r}+\sigma_{\theta}\right)+(1-2 v) \mathrm{P}_{\alpha}$

(4) $\frac{\partial \varepsilon_{\mathrm{vp}}}{\partial \mathrm{t}}=\frac{1}{\mathrm{n}}\left\langle\sigma_{\mathrm{r}}-\sigma_{\theta}-2 \mathrm{C}\right\rangle$

(5) $\frac{\partial \sigma_{r}}{\partial r}+\frac{\sigma_{\theta}-\sigma_{r}}{r}=0$.

\section{Remarques}

Les quatre premières équations traduisent les « relations de comportement $"$; les trois premières constituent des intégrales des relations en vitesses, compte tenu qu'à l'instant initial le massif est dans un état naturel. La troisième relation traduit l'hypothèse de déformation plane; on s'est placé dans le cas du régime de face, c'est-à-dire que, compte tenu de la nature du chargement, on suppose que l'ordre des contraintes principales reste le suivant (cette hypothèse limite un peu la généralité du calcul):

$$
\sigma_{r}>\sigma_{z}>\sigma_{8} \text {. }
$$

Dans la quatrième équation, qui n'est correcte que compte tenu de l'inégalité précédente, le symbole \langle\rangle signifie "partie positive de . Enfin la dernière relation traduit l'équilibre.

On vérifie facilement sur ces équations qu'il n'y a pas d'inconvénient à prendre la valeur de la cohésion C comme unité pour les contraintes et la quantité $2 \eta\left(1-v^{2}\right) / E$ comme unité pour le temps; on simplifie ainsi l'écriture des formules.

\section{Processus de résolution}

La relation (4) n'est utilisable que dans la zone viscoplastique; par contre, les autres relations permettent d'éliminer $\sigma_{e}, \sigma_{z}$ et $\varepsilon_{v p}$ pour obtenir une relation intégrable par rapport à $r$ qui est valable dans la totalité du massif :

(6) $E \frac{u}{r}=(1+v)(1-2 v)\left(\sigma_{r}+P_{\star}\right)-2\left(1-v^{2}\right) \frac{y^{2} \sigma_{y}}{r^{2}}$.

On a noté $\mathrm{y}^{2} \sigma_{y}$ la constante d'intégration; pour justifier cette notation, il est nécessaire de décrire l'évolution des phénomènes lorsqu'apparaît la viscoplasticité :

1) Lorsqu' on commence à charger la structure à partir de l'état naturel en augmentant la valeur du paramètre $\sigma_{i}$, le comportement est d'abord purement élastique, tant qu'est vérifiée la condition $\sigma_{r}-\sigma_{0}<2$. Puis, le chargement augmentant, apparaît d'abord à la paroi de la cavité une zone viscoplastique. On note alors $y^{2}$ le carré du rayon du cercle qui limite à l'intérieur la zone viscoplastique (caractérisée par $\varepsilon_{\mathrm{vp}} \neq 0, \frac{\partial \varepsilon_{\mathrm{vp}}}{\partial \mathrm{t}} \neq 0$ ) et à 


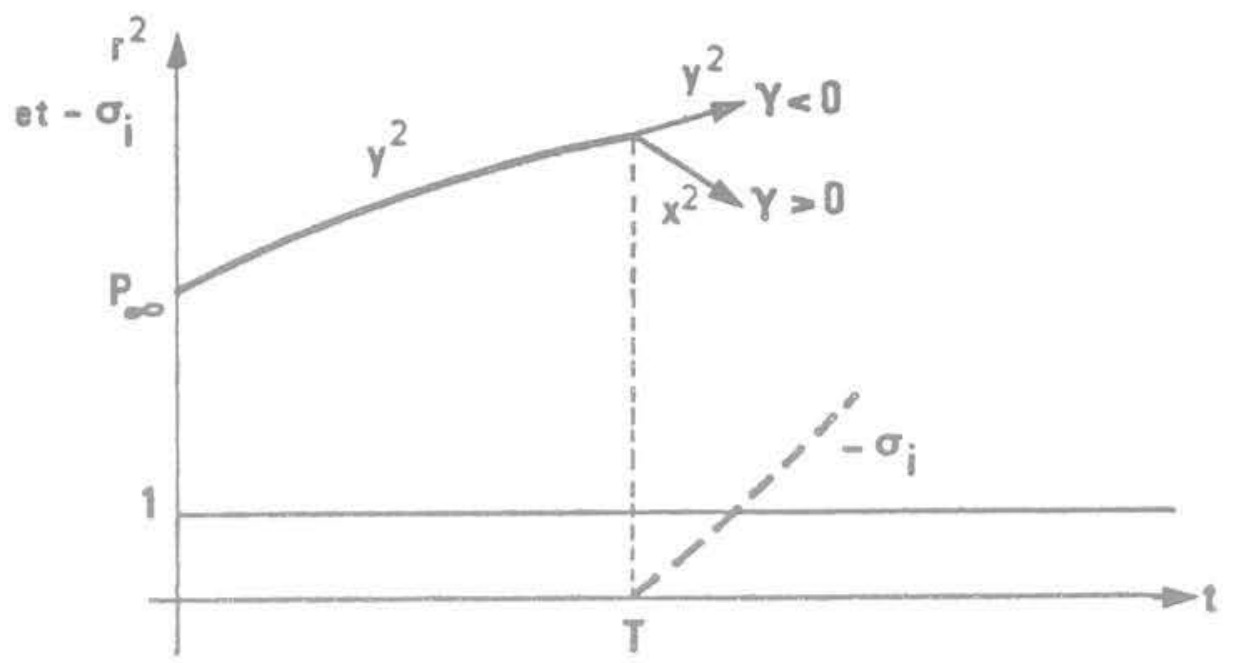

Fig. 2 Evolution des phénomènes après la pose du soutènement $(t=T)$, en fonction du facteur de rigidité $\gamma$ du revêtement

l'extérieur la zone élastique (caractérisée par $\varepsilon_{\mathrm{vp}}=0$ et $\partial \varepsilon_{\mathrm{vp}} / \partial \mathrm{t}=0$ ). On vérifie facilement que sur ce rayon y la contrainte radiale vaut 1 ; on notera :

$$
\hat{\mathrm{y}}^{2}>0: \sigma_{\mathrm{y}}=\sigma_{\mathrm{r}}(\mathrm{y})=1 \text {. }
$$

On démontre alors (Tijani, 1978, Bérest et Nguyen Minh D., 1982) que l'évolution de y est gouvernée par l'équation:

$$
\dot{\sigma}_{i}+\left(P{ }_{c c}+\sigma_{i}\right)=\dot{\vec{y}}^{2}+1+\log y^{2} .
$$

2) La formule précédente ne s'applique que tant que $\mathrm{y}^{2}>0$. Si l'on est conduit au cours du chargement à inverser le sens de l'évolution du paramètre $\sigma_{i}$, l'évolution du rayon de la zone viscoplastique peut également changer de sens. On conserve alors la notation y pour désigner l'extension maximale du rayon de la zone viscoplastique. On note $\mathrm{x}^{2}$ le carré du rayon du cercle qui limite la zone viscoplastique: on est ainsi conduit à distinguer trois zones dans le massif :

$y \leqslant r<\propto$ : zone élastique.

$$
\varepsilon_{\mathrm{vp}}=0 \text { et } \frac{\partial \varepsilon_{\mathrm{vp}}}{\partial \mathrm{t}}=0
$$

$\mathrm{x} \leqslant \mathrm{r} \leqslant \mathrm{y}:$ zone à déformation résiduelle,

$$
\varepsilon_{\mathrm{vp}} \neq 0 \text { et } \frac{\partial \varepsilon_{\mathrm{vp}}}{\partial t}=0
$$

$1 \leqslant r \leqslant x:$ zone viscoplastique,

$$
\varepsilon_{v p} \neq 0 \text { et } \frac{\partial \varepsilon_{v p}}{\partial t} \neq 0 \text {. }
$$

II est utile de noter que dans une telle phase $y^{2}$ est constant mais $\dot{\sigma}_{y}<0$, alors que dans la première phase décrite $\widehat{y}^{2}>0$, mais $\sigma_{y}=1$.

$t$ désignant l'instant actuel où le rayon de la zone viscoplastique est en $\mathrm{x}$, on désigne par $\mathrm{t}_{c}$ l'instant où, dans la première phase, le rayon viscoplastique passait par le même point:

$$
y^{2}\left(t_{c}\right)=x^{2}(t)
$$

On démontre alors (Bérest et Nguyen Minh Duc, 1982) que l'évolution de $\mathrm{x}^{2}$ est décrite par l'équation :

(8) $\frac{d x^{2}}{d t}(t)=\frac{d y^{2}}{d t}\left(t_{c}\right)-\dot{\sigma}_{i}\left(t_{c}\right)+\dot{\sigma}_{i}(t) \exp \left(t-t_{c}\right)$.
On a de plus la formule utile :

$$
\frac{d x^{2}}{d t}(t)=\exp \left(t-t_{0}\right) \frac{d}{d t}\left(y^{2} \sigma_{y}\right) .
$$

Comme les quantités relatives à l'instant $t_{c}$ sont connues par le calcul de l'évolution de $\mathrm{y}^{2}$ pendant la phase précédente, (8) permet de calculer l'évolution de $x^{2}$.

La justification et l'intérêt de la notation $y^{2} \sigma_{y}$ est ainsi explicitée et on peut appliquer les formules (6 à 9) à notre cas particulier.

\section{Application au problème du tunnel}

\section{A. Évolution avant la pose du revêtement}

On a supposé qu'à l'instant initial on appliquait une contrainte radiale nulle à la paroi : $\sigma_{i}(t)=-P_{\infty}(1-Y(t))$ où $Y(t)$ désigne la fonction Heaviside. De l'équation (7) on déduit que $\mathrm{y}^{2}$ subit initialement un saut égal à $+\mathrm{P}_{\infty}$ : puis que l'évolution ultérieure est régie par

$$
\frac{d}{d t} y^{2}=P_{\infty}-1-\log y^{2}
$$

ou encore :

$t \exp \left(1-P_{x}\right)=E_{i}\left(P_{x}-1-\log P_{x}\right)$

$$
-E_{1}\left(P_{x}-1-\log y^{2}\right) \text {. }
$$

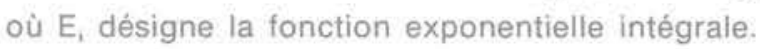

$\mathrm{y}^{2}$ tend donc vers une valeur asymptotique égale à la valeur qui serait obtenue pour un comportement élastoplastique non visqueux :

$$
y_{F}^{2}=\exp \left(P_{\infty}-1\right) \text {. }
$$

Et le déplacement à la paroi s'écrit dans cette phase :

$$
\operatorname{Eu}(t, 1)=-2\left(1-v^{2}\right) \mathrm{y}^{2}(\mathrm{t})+(1+v)(1-2 v) \mathrm{P}_{\alpha} .
$$

B. Pose du revêtement

A l'instant $T$, on pose un revêtement élastique; l'évolution de la convergence sera alors modifiée par l'apparition d'une contrainte normale appliquée par le revêtement au massif qui est proportionnelle à 


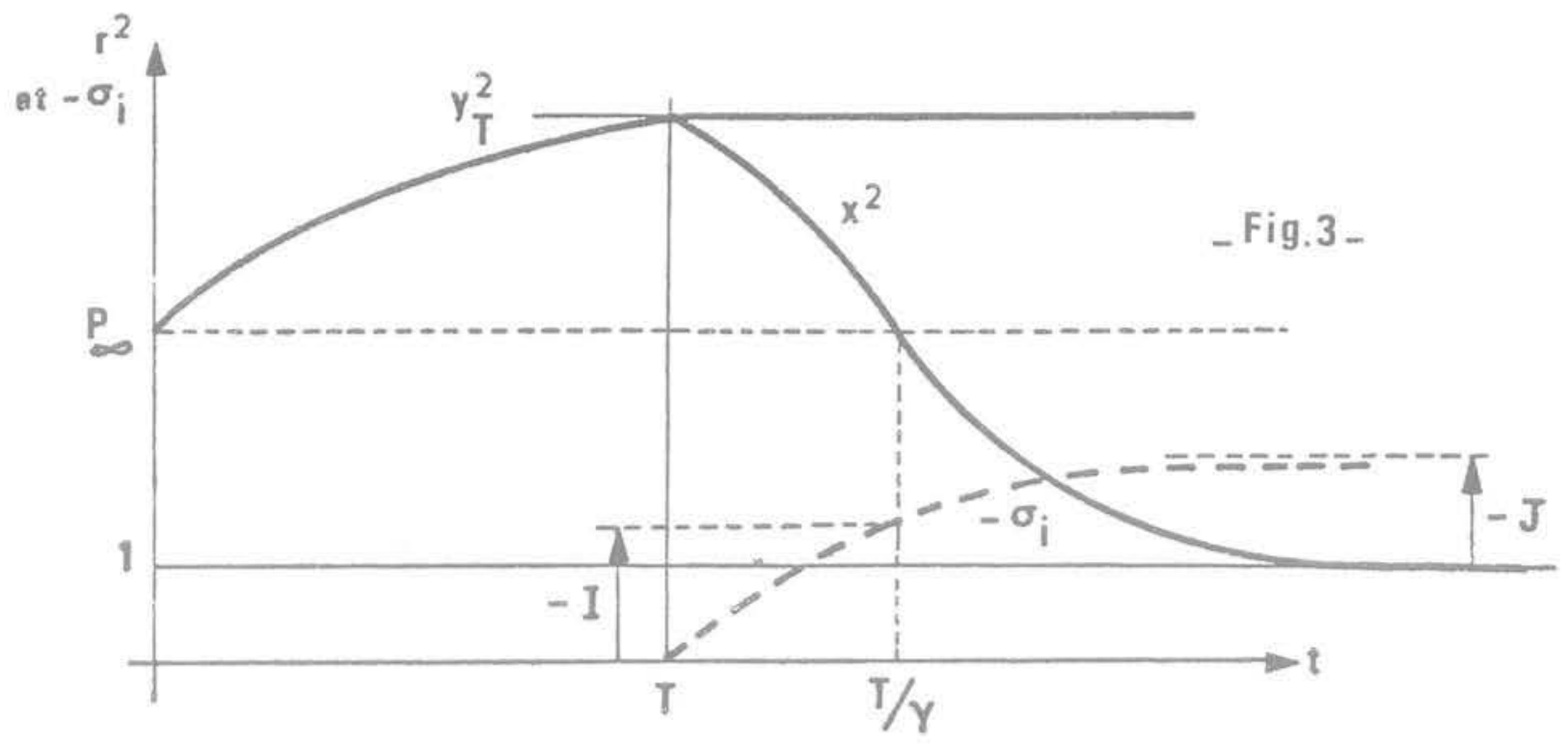

Fig 3 Revêtement raide : allures des phénomènes et notations

I accroissement de la convergence :

$$
G[u(t, 1)-u(T, 1)]=s_{i}(t)-s_{i}(T) .
$$

oủ $S_{i}(T)$ est nul et $G$ est la « raideur d du revêtement.

Compte tenu de (6), on a :

$$
\begin{aligned}
& \gamma \dot{s}_{i}=\frac{d}{d t}\left(y^{2} \sigma_{y}\right) \\
& \gamma=\frac{1-(1+v)(1-2 v) \frac{G}{E}}{-2\left(1-v^{2}\right) \frac{G}{E}} .
\end{aligned}
$$

On vérifie que $v$ étant compris entre 0 et $0,5, \gamma$ peut varier entre $-x$ et 0,5 .

Comme $\dot{s}_{i}$ est nécessairement négatif, le signe de $\frac{\mathrm{d}}{\mathrm{dt}}\left(\mathrm{y}^{2} \sigma_{\mathrm{v}}\right)$ dépend de celui de $\gamma$.

\section{Cas du revêtement " mou"}

$\gamma$. 0 : le revêtement est " mou " et $\frac{d}{d t}\left(y^{2} \sigma_{y}\right)=\frac{d y^{2}}{d t}>0$; la zone viscoplastique continue à croitre suivant la loi :

$$
\left(\frac{1}{y}-1\right) \hat{\mathrm{y}}^{2}+\frac{\mathrm{y}^{2}-\mathrm{y}_{\mathrm{T}}^{2}}{\gamma}-1-\log \mathrm{y}^{2}+\mathrm{P}_{\mathrm{x}}=0
$$

$$
\begin{aligned}
E u(t, 1)=\frac{1}{\gamma}(1+v)(1-2 v) \\
\quad\left(\mathrm{y}^{2}-\mathrm{y}_{T}^{2}+\gamma \mathrm{P}_{\infty}\right)-2\left(1-v^{2}\right) \mathrm{y}^{2} .
\end{aligned}
$$

II est facile de vérifier sur ces formules que $y^{2}$ tend au bout d'un temps infini vers $y_{F}^{2}$ défini par:

$$
\frac{y_{F}^{2}-y_{T}^{2}}{\gamma}-1-\log y_{F}^{2}+P_{x}=0
$$

Et que la contrainte normale appliquée par le revêtement au massif sera après un temps infini :

$$
\sigma_{F}=\frac{1}{\gamma}\left(y_{F}^{2}-y_{T}^{2}\right) \text {. }
$$

II est à noter que les valeurs finales du rayon de la zone plastique et de la pression de soutenement sont les mêmes que si le comportement était élastoplastique non visqueux (voir Annexe).

D. Cas du revêtement " raide" $\gamma>0$ : le revêtement est "raide " et

$$
\frac{d}{d t}\left(y^{2} \sigma_{y}\right)=y_{T}^{2} \frac{d \sigma_{y}}{d t}<0 ;
$$

la zone viscoplastique voit son rayon diminuer; il faut alors utiliser les formules (8) et (9). Dans cette phase de décharge, on distinguera pour l'application de ces formules deux périodes suivant la place de $x^{2}$ par rapport à $P_{\times}$. Mais, on peut dans tous les cas déduire des formules (9) et (11) la relation suivante :

(13) $\dot{\sigma}_{i}(t) \exp \left(t-t_{c}\right)=\frac{1}{\gamma} \frac{d}{d t}\left(y^{2} \sigma_{y}\right) \exp \left(t-t_{c}\right)=\frac{1}{\gamma} \frac{d x^{2}}{d t}$. rappel figure 3

a) Lorsque $y_{T}^{2} \geqslant x^{2} \geqslant P_{x}$ on doit faire dans $(8) \dot{\sigma}_{i}\left(t_{c}\right)=0$ et donc:

$$
\left(1-\frac{1}{\gamma}\right) \dot{\bar{x}}^{2}(t)=\dot{\hat{y}}_{\mathrm{T}}^{2}\left(t_{c}\right)
$$

On en déduit que $x^{2}=x^{2}(t)$ est transformée par affinité de rapport $\left(1-\frac{1}{\gamma}\right)$ et d'axe vertical $t=T$ de la courbe $\mathrm{y}^{2}=\mathrm{y}^{2}\left(\mathrm{t}_{\mathrm{c}}\right)$; cette phase s'achève donc à l'instant $\mathrm{T} / \gamma$; à cet instant, la contrainte $I=\sigma_{i}(T / \gamma)$ appliquée par le revêtement au massif est donnée par l'intégration de (13) :

$$
\begin{aligned}
\mathrm{I=} \sigma_{i}\left(\frac{T}{\gamma}\right)=\int_{T}^{T / \gamma} \frac{1}{\gamma} \exp \left(t_{c}-t\right) \frac{d x^{2}}{d t} d t & =\int_{0}^{T} \\
& -\frac{1}{\gamma} \frac{d y^{2}}{d t_{c}} \exp \frac{T-t_{c}}{\gamma} d t_{c} .
\end{aligned}
$$

La deuxième intégrale étant obtenue par le changement de variable $t=t_{c}+\frac{T-t_{c}}{\gamma} ; I=I(T)$ est alors sous la forme d'une convolution:

$$
I(0)=0 ; \quad \frac{d l}{d T}+\frac{1}{\gamma} I+\frac{1}{\gamma} \frac{d y^{2}}{d t}(T)=0 .
$$



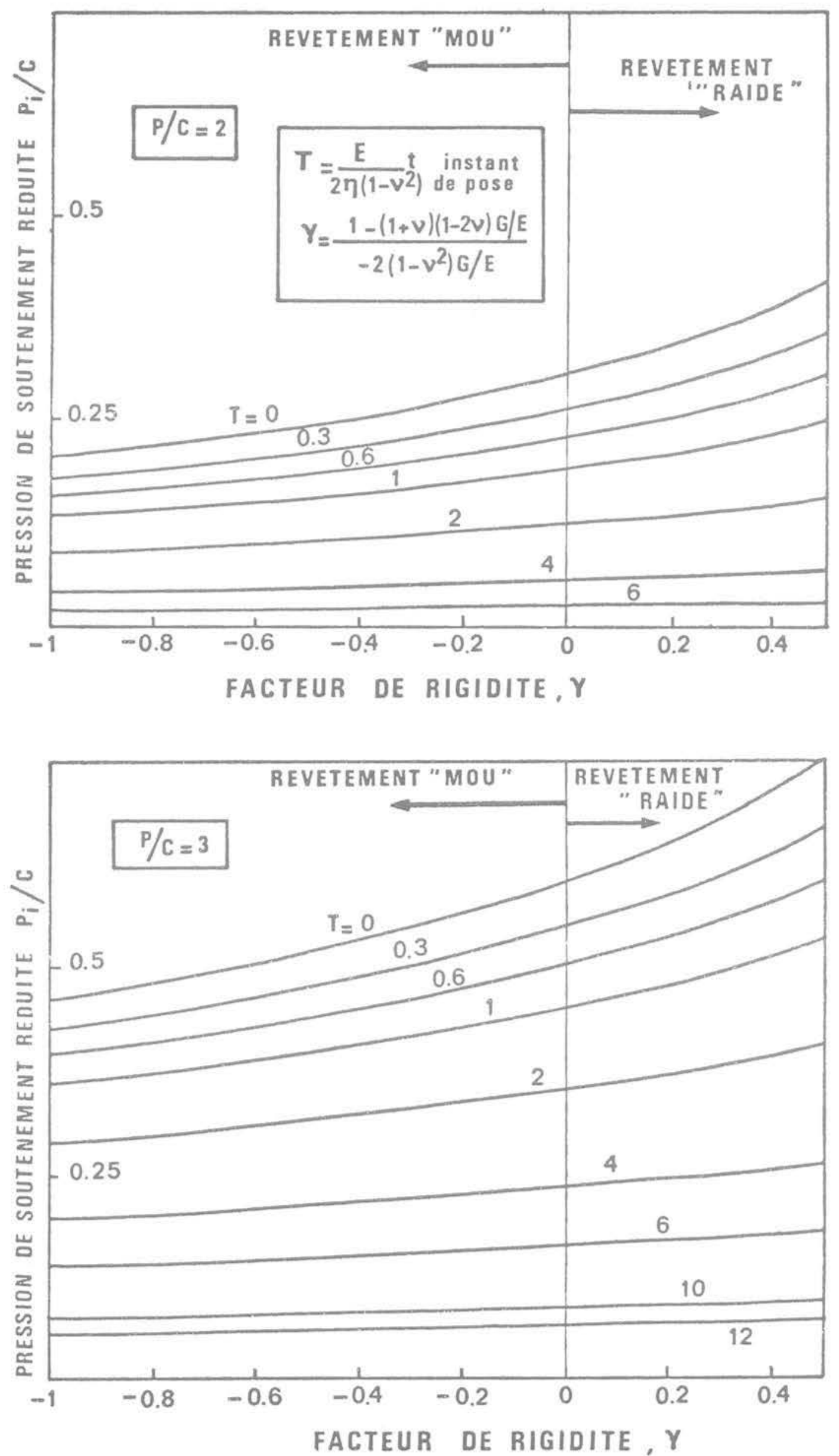

Fig. 4 Abaques donnant la pression de soutènement en fonction du facteur de rigidité $\gamma$ du revêtement pour différents instants de pose 
II est plus commode pour le calcul de considérer I comme une fonction de $\mathrm{y}^{2}$ dont on calcule la valeur au point $\mathrm{y}^{2}=\mathrm{y}_{\mathrm{T}}^{2}$ :

$$
I\left(P_{\infty}\right)=0 ; \quad \frac{d l}{d y^{2}}+\frac{1}{\gamma\left(P_{\infty}-1-\log y^{2}\right)}+\frac{1}{\gamma}=0 .
$$

b) Lorsque $P_{\infty} \geqslant x^{2} \geqslant 1$, l'instant $t_{c}$ est l'instant initial. pendant lequel est vérifiée la relation:

$$
\frac{d y^{2}}{d t}\left(t_{c}\right)-\dot{\sigma}_{i}\left(t_{c}\right)=y^{2}-1-\log y^{2} .
$$

De sorte que la formule (8) conduit à la relation :

$$
\left(1-\frac{1}{\gamma}\right) \frac{d x^{2}}{d t}=x^{2}-1-\log x^{2},
$$

$\mathrm{x}^{2}$ tend donc asymptotiquement vers la valeur 1 : la zone viscoplastique s'évanouit après un temps infini, le comportement étant de l'ordre de $x^{2}-1 \sim \frac{2(1-1 / \gamma)}{t}$. La contrainte normale appliquée après un temps infini par le revêtement au massif sera donc:

$$
\sigma_{F}=1+J=1+\int_{T / \gamma}^{\infty} \frac{1}{\gamma} \exp (-t) \frac{d x^{2}}{d t} d t,
$$

I a été calculé précédemment; pour calculer $\mathrm{J}$, on pose $\mathrm{K}=\mathrm{J} \exp (\mathrm{T} / \gamma)$ de sorte que $\mathrm{K}$ prend la forme d'une convolution :

$$
K(\infty)=0 ; \quad \frac{d K}{d(T / \gamma)}-K+\frac{1}{\gamma} \frac{d x^{2}}{d t}(T / \gamma)=0 .
$$

Comme précédemment, il est commode de considérer $\mathrm{K}$ comme une fonction de $\mathrm{x}^{2}$ dont on calcule la valeur lorsque $\mathrm{x}^{2}=\mathrm{P}_{\infty}$ :

$$
\mathrm{K}(1)=0 ; \quad \frac{\mathrm{dK}}{\mathrm{d} \mathrm{x}^{2}}-\left(1-\frac{1}{\gamma}\right) \frac{\mathrm{K}}{\mathrm{x}^{2}-1-\log \mathrm{x}^{2}}+\frac{1}{\gamma}=0 .
$$

II est bien entendu à remarquer que dans le cas du revêtement " raide $"$ la pression de soutènement finale calculée n'est pas la même que si le comportement était élastoplastique non visqueux.

\section{E. Conclusion du calcul et applications numéri- ques}

On a pu mettre en évidence une distinction fondamentale entre revêtement " mou " et revêtement " raide " tels qu'ils ont été définis dans le texte: le premier induit une augmentation de la zone viscoplastique, qui converge vers la valeur obtenue dans un calcul élastoplastique sans viscosité; au contraire, le revêtement "raide" provoque une régression de la zone viscoplastique; l'état final obtenu est distinct de celui donné par une solution élastoplastique sans viscosité.

La figure 4 donne des abaques représentant l'évolution en fonction du paramètre $\gamma$ de la pression finale exercée sur le revêtement, pour différents instants de pose T. L'intégration numérique a été faite par la méthode de Treanor à pas variable (J. Zarka, 1980). 


\section{Annexe}

Comparaison avec la méthode "convergence-confinement"

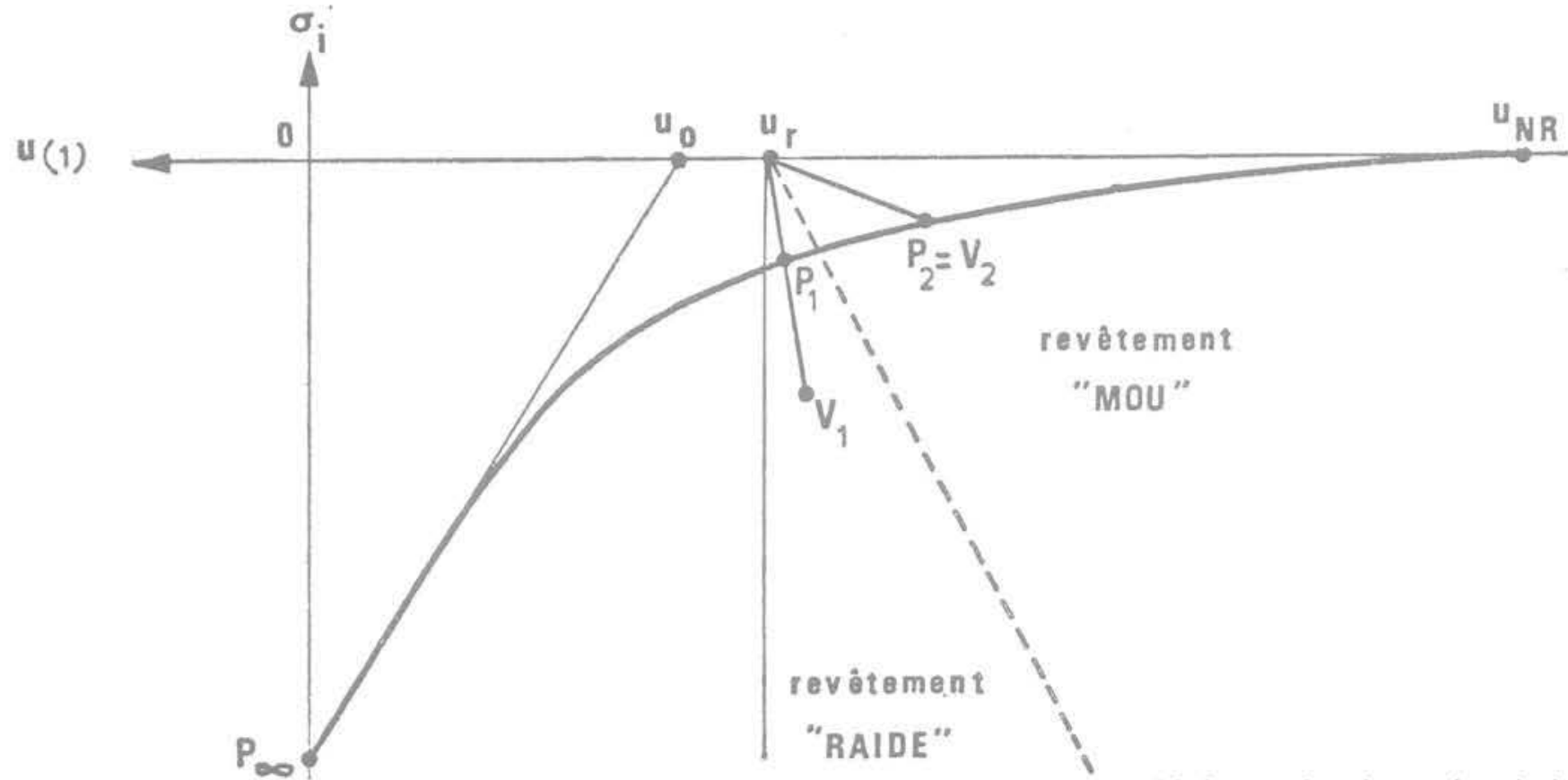

Fig. 5 Comparaison des résultats du calcul élastoviscoplastique avec la "courbe caractéristique», donnée par le calcul élastoplastique

A différentes étapes du calcul, on a comparé les résultats obtenus avec ceux que donne le modèle élastoplastique sans viscosité.

Dans ce dernier, l'évolution de la convergence u(1) en fonction de la contrainte normale appliquée $\sigma_{i}$ est donnée par les relations

${ }^{*} \sigma_{i}+\mathrm{P}_{\infty} \leqslant 1: \operatorname{Eu}(1)=-(1+v)\left(\sigma_{4}+\mathrm{P}_{\infty}\right) ;$

${ }^{*} \sigma_{i}+\mathrm{P}_{\infty} \geqslant 1 ; \mathrm{Eu}(1)=(1+v)(1-2 v)\left(\sigma_{i}+\mathrm{P}_{\alpha}\right)$

$$
-2\left(1-\nu^{2}\right) \exp \left(\sigma_{i}+P_{\infty}-1\right) \text {. }
$$

La courbe représentative de cette évolution est dite " courbe caractéristique du terrain ". Bn admet dans la méthode "convergence-confinement " qu'en posant à l'instant $T$ caractérisé par une convergence $u_{T}$ un revêtement élastique on atteindra l'équilibre au point $P$, intersection de la courbe caractéristique du terrain avec la courbe caractéristique du revêtement

$$
\sigma_{\mathrm{i}}=\mathrm{G}\left(\mathrm{u}-\mathrm{U}_{\mathrm{T}}\right) \text {. }
$$

II faut noter que la méthode "convergenceconfinement " repose sur l'interprétation d'un phénomène que nous avons délibérément négligé: dans cette méthode, c'est l'éloignement progressif du front qui explique que la courbe caractéristique du terrain soit progressivement décrite; si le tunnel était instantanément creusé, on " sauterait " immédiatement au point $U_{N A}$ caractéristique de la convergence finale d'un tunnel non revêtu.

Bien entendu, on sait que dans la réalité on ne doit pas tenir compte seulement de ce phénomène, mais aussi de la viscosité du massif; mais, en l'absence de modèle tenant compte de la viscosité, on est contraint de considérer que son effet est englobé dans la notion de mise en charge progressive par le front.

Dans le calcul présenté ici, on n'a au contraire pas tenu compte de l'effet du front et on s'est limité au seul effet de la viscosité du massif : il est remarquable que pour les revêtements dits "mous", les deux méthodes donnent des résultats identiques, mais que pour les revêtements dits "raides " la pression de revêtement finale soit supérieure (quoique d'assez peu dans les applications à des cas vraisemblables) à celle donnée dans la méthode "convergence-confinement ".

II est certain qu'une interprétation meilleure des phénomènes qui affectent une galerie réelle résultera de la prise en compte simultanée de ces deux aspects du comptement différé: l'introduction de l'effet du front dans le calcul viscoplastique ne pose en effet aucun probleme de principe de sorte qu'une des limitations de la méthode classique de "convergenceconfinement " sera ainsi levée.

\section{Références}

M. Panet, 1979, "Les déformations différées dans les ouvrages souterrains", C. R. du $4^{\mathrm{e}}$ Congrès International de la S.I.M.R., Montreux. Tome 3, pp. 291-301.

Tunnels et Ouvrages souterrains, $n^{\circ} 32$, Mars-Avril 1979, pp. 70-76, a Stabilité des tunnels par la méthode 'convergence - confinement' ». (Rapport général sur la journée d'Études de l'AFTES, 26.10.78, Paris).

S. M. Tijani, 1978, "Résolution numérique des problèmes d'élastoviscoplasticité. Application aux cavités de stockage du gaz en couches salines profondes ", thèse Docteur-Ingénieur.

P. Berrest, Nguyen Minh D., 1982, "Response of a spherical cavity in an elastic viscoplastic medium submitted to a variable internal pressure ", à paraitre dans Int. J. of Solids et Structures.

J. Zarka, 1978, «Principe des méthodes actuelles de résolution numérique des problèmes classiques en Mécanique des Solides." Cours E.N.S.T.A. 